\title{
Assessment Of Emerging Cooling Technologies By Analyzing Their Impact On Reducing The Power Usage Effectiveness Ratio Of Data Centers
}

\author{
Omkar Gadgil ${ }^{\mathrm{a}}$, Keith A. Rahn ${ }^{\mathrm{b}}$ \\ ${ }^{a}$ Auburn University, 118 M. Miller Gorrie Center, Auburn, 36849, USA \\ ${ }^{b}$ Auburn University, 222 M. Miller Gorrie Center, Auburn, 36849, USA
}

\begin{abstract}
With the advent of high speed internet, smart-phones, Wi-Fi, Cloud computing, and IOT, our desire for creating and consuming data has been growing exponentially. It has resulted in great demand for storing and processing of this data with increase in number and scale of data center facilities around the globe. Data centers have started consuming much higher amount of power and highperformance IT equipment have presented a bigger challenge for cooling systems. Large technology companies have realized the need for making their data centers more energy efficient and, in turn, modern mechanical systems have been designed to work more efficiently. This research study endeavors to analyze these mechanical systems and present the way they affect Power Usage Effectiveness (PUE) ratios of data centers. Data from numerous data center projects carried out by a leading general contractor in last 5-years was obtained and correlation analysis was carried out to assess how modern mechanical systems help in reducing their PUE ratios.
\end{abstract}

(C) 2019 The Authors. Published by Budapest University of Technology and Economics \& Diamond Congress Ltd.

Peer-review under responsibility of the scientific committee of the Creative Construction Conference 2019.

Keywords: data; effectiveness; mechanical; power; usage

\section{Introduction}

One of the biggest technology revolutions the last decade brought with it was 'Big-Data' [1]. With the advent of high speed internet, smart-phones, Wi-Fi, Cloud computing, and IOT, our desire for creating and consuming data has been growing exponentially. This means a lot more space is required to store this data. Those days of having only a server room in your building to store and process this data are long gone and a new category of buildings called 'Data Center' has been established. In last 10-years, we have seen unprecedented growth in data center projects all over the world. This growth is observed both in numbers and scales of data center projects. Technological advancements in servers and other IT equipment have made these machines more compact with capabilities of handling highly complex data processing and transfer very efficiently; giving rise to higher power consumption and heat output in the same confined rack space.

Last decade also brought a lot of awareness about energy efficiency. Some of the main reasons for that were rising power costs, carbon emissions, and global warming. They have made the construction industry realize the importance of building structures that consume less energy and function more efficiently. Data Centers are one of the highest

*Keith A. Rahn email: kar0023@auburn.edu 
power consuming buildings for their size [2]. Hence, a significant amount of research is being done to reduce their overall energy needs. Mechanical cooling systems, being the largest user of energy amongst all non-IT equipment, have become the obvious choice for improving efficiency of a data center. The Green Grid, a non-profit organization specializing in data center research, introduced and promoted a metric called PUE (Power Usage Effectiveness) for measuring efficiency of data centers in 2006-07. Since then, PUE has become a globally accepted metric for establishing energy performance.

\section{Literature review}

The main purpose of mechanical systems in data centers is to maintain suitable environmental conditions around server racks within acceptable limits so that the heat generated from various IT equipment inside the racks can be dissipated keeping them cool. Continuous and reliable functioning of these mechanical systems is critical for any data center operation. Heating, Ventilation and Air Conditioning (HVAC) systems used in data centers that were constructed in early 2000s have been using the traditional method (Figure 1) of blowing cold air in the area which houses the IT equipment. With the development of higher power consuming IT equipment, resulting from fitting more and more transistors onto chips inside the servers and creating denser equipment, we have seen tremendous increase in rack level power use. An IT equipment rack (more commonly known as Server Rack) is a metal enclosure (cabinet) which houses various IT equipment such as servers, switches, storage devices and is usually connected to a power source above or below the cabinet. Within last 10 to 15 years the power consumption capacities of server racks have increased from 5 kilowatts $(\mathrm{kW})$ per cabinet up to $25 \mathrm{~kW}$ and are expected to reach $50 \mathrm{~kW}$ in the next few years.

\section{Traditional Cooling Diagram}

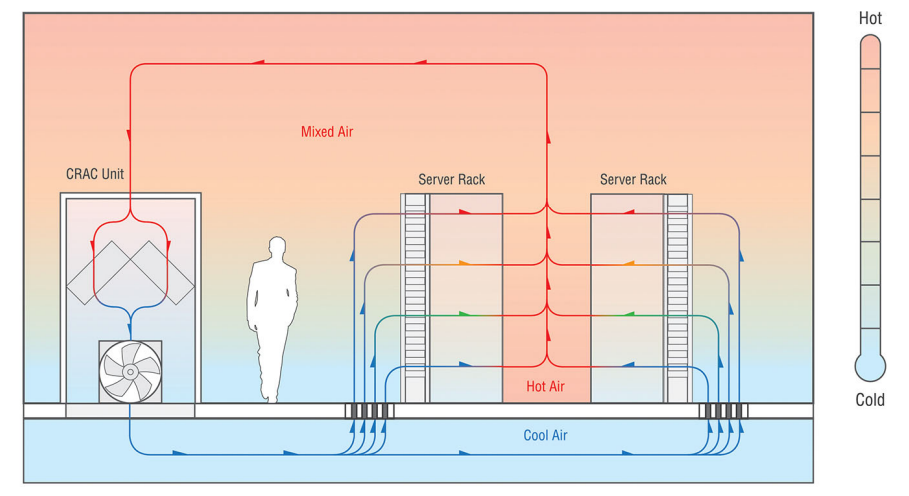

Figure 1: Traditional Data Center cooling system using raised floors.

Source: https://journal.uptimeinstitute.com/wp-content/uploads/2015/07/N-Sasser-Figure-3image2.jpeg

Free Cooling, as the name suggests, is a mechanical system that uses colder outside weather to directly cool the IT equipment space. The medium of heat transfer in this case is air, like the traditional cooling systems. However, there are two broad methods of using colder climatic conditions to supply cold air to IT rack space. First, is to directly take outside cold air and second is to use water, cooled by outside cold atmosphere. While designing and implementing this system, the engineers are first expected to ensure that the air flow management inside the data center is designed with utmost care. One of the ways to improve the air-flow and to ensure minimum mixing of hot and cold air would be to use Hot-aisle containment system. This system uses an enclosure to trap and remove hot air coming out of the rear side of racks. This stops the flow of hot air from mixing with cold air being pumped in the IT equipment space.

Direct free cooling allows outside air to pass through a series of filters in the air handling unit (AHU) before getting pumped in the IT equipment space. A significantly large assembly of filters is required to be installed for the outside air to get purified in order to maintain the indoor air quality. As this system is used in colder climates, concentration of pollutants and particulate matter is higher in cold conditions than warmer.

The second method of free cooling, indirect air-side free cooling, does not allow the outside air to mix with indoor air. A well-known example of this concept is Kyoto Wheel system [3]. It operates through air-to-air heat exchanger, which 
allows transfer of heat on a rotary heat wheel. One of the biggest advantage of indirect air-side free cooling is that it allows to maintain desirable indoor air quality (IAQ) by keeping away the pollutants and particulate matter from outside air.

IT equipment racks in data centers have different capacities of power consumption ranging from 1 or $2 \mathrm{~kW}$ to more than $25 \mathrm{~kW}$. Higher power consumption capacities mean those racks have higher density of servers which result in to more heat output in a confined space. When these IT equipment racks start getting denser, air as a medium of heat transfer, starts becoming inefficient. In this situation Liquid Cooling, which uses water or other engineered liquids (aka Liquid Coolants) as a medium of heat transfer needs to be employed [4]. Liquid coolants are known to have better thermal properties than air. Their thermal conductivity, specific heat, and density are higher as compared to air [5].

One of the main advantages of using liquid cooling is that the temperature of liquid coolant required to be supplied to the racks is considerably higher than the temperature of air pumped in to the rack space when using conventional systems [6]. Therefore, less amount of energy is required to operate the chillers, reducing the overall energy consumption of the mechanical systems.

Liquid Immersion Cooling is the latest cooling technology which is gaining popularity in the data center industry [4]. In this system, the IT equipment is immersed in a dielectric (non-conductive) liquid such as 3M's NOVECTM 649. The engineered fluid changes its state from liquid to gas when it absorbs heat from high-heat generating components inside the IT equipment immersed in the tank. This gas being less dense than the liquid rises to the space above the liquid. It is then taken out into a condenser which is cooled by either chilled water or another refrigerant. Through this cycle, the gas cools down, regains its liquid state and travels back to the rack space [7]. One more advantage of using liquid immersion cooling, apart from energy savings from higher allowable inlet temperature of coolant, is considerable fan energy savings and lower noise levels [4].

Direct evaporative cooling is similar to that of free cooling in configuration but totally different conceptually. Direct evaporative cooling is used in places where outside temperature is not suitable for free cooling. This cooling system is also called as adiabatic cooling which uses the cooling effect of water as it evaporates [8]. Direct evaporative cooling became Google, Facebook, Microsoft and other large technology companies' choice for their data center projects [9]. Since then, Facebook has been using DEC system on their data centers and has shown significant reduction in water usage by implementing cooling strategies specific to each location [10].

The Green Grid is a non-profit, open industry consortium of information and communications technology (ICT) industry professionals that works to improve IT and data center resource efficiency around the world. It was founded in year 2007 and right after their inception they presented the idea of Power Usage Effectiveness (PUE) metric. As simple as it sounds, this metric is a ratio of total energy required by a data center facility and the amount of energy required by the IT equipment.

$$
\text { P. U. E. }=\frac{\text { Total Facility Energy }}{\text { IT equipment Energy }} \times 100 \%
$$

Based on the PUE formula, we can say that achieving PUE ratio of 1.0 is the best possible scenario for any data center facility. This means, no additional power is required to run the facility apart from the IT equipment. Though this scenario sounds like a utopian concept, large data center operators like Google have managed to bring down this ratio very close to 1.0 [11]. The Green Grid and Uptime institute have been recording PUE ratios of various data centers since 2007. Their initial data shows that on average the PUE ratios in year 2007 were close to 2.50 which significantly improved to 1.89 in year 2011 but by year 2014 it showed little improvement as the average reported PUE ratio was 1.7 [12]. The Environmental Protection Agency (EPA) has defined a PUE ratio of 1.5 as a 'best practice' target from year 2011 (Figure 2) for the data center industry [13]. Hence, one of the main objectives of this research was to find out if by use of emerging mechanical systems, the PUE ratios have improved in last 5-years. 
Keith A. Rahn / Proceedings of the Creative Construction Conference (2019) 088 https://doi.org/10.3311/CCC2019-088

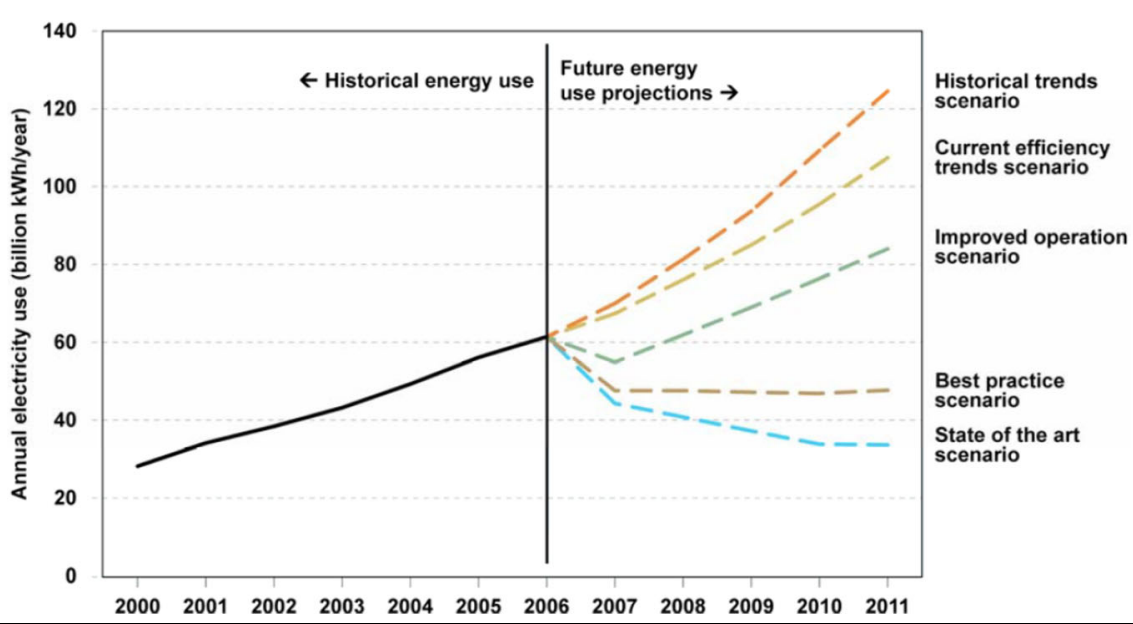

Figure 2: Comparison of projected electricity use by Data Centers (2007 to 2011) and best practice scenarios. Source: Report to Congress on Data Centers Energy Efficiency - Public Law 109-431, U.S. Environmental Protection Agency, Energy Star Program, August 2, 2007.

Weather conditions play an important role in energy consumption of the buildings. It can be easily understood that the PUE ratio of a facility will be higher in the hotter months of the year and lower in the winter. Researchers at University of Leeds, UK provided a critical analysis of the PUE metric which outlined the type of detailed engineering data required to obtain meaningful values of PUE [14]. Another study carried out at Stanford [15] showed the difference in PUE values for a hypothetical data center in 2 different cities and recommended that including weather/climate information while calculating the PUE ratios would provide a better understanding of energy efficiency.

Many operators calculate and report their facilities' PUE ratios as average of a year. Moreover, due to geographical locations of the data center facilities, the environmental conditions make significant impact on average yearly PUE values. For example, if all other factors such as infrastructure size, type, cooling technology etc. are considered equal, average PUE ratios of data centers in places like Arizona, Georgia or Florida would theoretically be higher than the facilities in Indiana or Connecticut.

A data center facility may or may not use only electricity as its soul energy source. Some facilities operate with multiple sources of energy such as electricity, natural gas, chilled water etc. Hence, in order to convert other sources of energy into electrical units - kWh, the Green Grid recommends using U.S. Source Energy Weighing Factors. To express total source energy, each fuel should first be converted into a single common unit $(\mathrm{kWh})$ and then multiplied by its weighting factor, after which the source energy for all fuels can be summed together [16]. The PUE ratios mentioned in the data received for analysis are expected to have considered this conversion in calculating their power consumption of the total facility.

The PUE metric has also been critically analyzed by some researchers. Energy consumption calculations for PUE ratios are sometimes based on engineering estimates which consider full-load name-plate ratings of infrastructure components to determine efficiencies. However, if equipment is under loaded, their efficiencies decrease considerably. So, it is recommended that PUE calculations shall be based on metered data during operations [15]. PUE calculations do not consider the efficiency of IT equipment such as server configurations, their productivity, energy losses due to server idling etc. [14]. Moreover, PUE ratios are being falsely interpreted for 'Greenness' as they neither consider the sources of energy nor the carbon emissions from the facility [17]. Another important factor to be considered while ensuring accuracy of the PUE calculations, as previously mentioned, is climatic conditions. Energy consumption of IT and non-IT equipment should be recorded over a longer time span, preferably a full year. "A comparison of data centers that includes climate information and data analysis alongside the PUE would give a more comparable ranking of energy efficiency" [14].

\section{Research}

The design of this research is based on the research onion described by Saunders and Lewis in their book "Doing Research in Business and Management" [18]. In the first layer, we have adopted the philosophy of realism. Like other 
scientific enquiries, the objectivity of this research suggests that the data exists independently of our knowledge of its existence. In the next layer, we have established the approach as deductive. The author has attempted to deduce a correlation between two factors related to data center facilities viz. mechanical technologies and PUE ratios.

Layer three strategy adopted by this research falls between a survey and a case-study approach. While the data about various data center facilities was collated using the survey approach, the analysis of this data was carried out using case-study approach. The methodology adopted for this study is based on quantitative research. The data required for performing correlation analysis was based on facts and figures. Hence, quantitative research methodology was considered as the best strategy. This study focused on emerging mechanical systems, the time horizon for analyzing the data was selected as last 5-years.

Data collection parameters that were established after extensive literature review. The author acknowledged that identity of most of the data center facilities would be kept confidential. Accordingly, those parameters such as project name or exact location were kept optional. The primary objective of requesting information on locations of the facilities was to establish their climatic zones. Other parameters like PUE ratios or types of mechanical systems were considered necessary for conducting a correlation analysis.

\section{Discussion}

As the traditional air conditioning equipment, also known as Computer Room Air Conditioners (CRACs) or Computer Room Air Handlers (CRAHs), used cold air to cool the racks. They used the volume of space under raised floors as plenums. Perforated grills on the raised floor were then used to supply this cold air up to the racks which, after passing through the servers, became hot and got mixed with the room air and went back to the CRAC machines for cooling again. Soon after data centers started becoming bigger and denser, these traditional mechanical systems were considered as inefficient due to flaws like uneven cooling, losses as well as space and design limitations [5].

While designing the mechanical systems in last few years, engineers have tried to address most of these shortcomings that the traditional cooling methods have. Especially for large data center facilities, a slight improvement in cooling efficiency makes a big impact due to the scale at which equipment perform. Initial efforts by design engineers to improve cooling efficiencies have yielded to improvements in air-flow management within the hot/cold aisles of racks. However, in recent years some engineers have tried to change the medium of heat transfer from air to liquid, while others have tried to make use of outside cold air in favoring climatic conditions to cool the server rack space.

\section{Conclusion}

'Big Data' has made a tremendous impact on the data center industry since year 2012. We also identified that IT equipment racks, being equipped with faster processors, switches, and higher capacity storage devices, now consume large amounts of power and in turn have higher heat output in the same confined spaces. This has given rise to modern cooling technologies which now focus more on energy efficiency. Power Usage Effectiveness (PUE) is a globally accepted metric for establishing the efficiency of a data center facility by calculating the ratio of total facility energy consumption and consumption by critical IT equipment.

Data center mechanical systems that use emerging cooling technologies are evolving and getting attention of engineers and owners, especially in the large data center construction market. Free Cooling Systems, which use simple fans and small pumps consume considerably lower amount of energy, have become more popular in last 5-years. They have helped larger and denser data centers in bringing down their PUE ratios significantly closer to 1.0. Average PUE ratios have shown steady decline in their values since year 2007. However, reduction in the average PUE ratios from 2011 to 2014 was not significant. Our analysis of the data from 40 data center facilities shows that facilities using traditional mechanical systems (using compressors, refrigerants, pumps, chillers etc...) have their PUE ratios in the range of 1.5 which match EPA's Best Practice target values. Use of modern mechanical systems on large data center facilities in the United States has significantly helped in bringing down their PUE ratios closer to 1.0

\section{References}

[1] Harvard Business Review, 2012 - https://pdfs.semanticscholar.org/02c7/740af5540f23a2da23d1769e64a8042ec62e.pdf

[2] FEMP, 2016 - https://datacenters.lbl.gov/sites/default/files/DataCenterEnergyReport2016_0.pdf 
Keith A. Rahn / Proceedings of the Creative Construction Conference (2019) 088 https://doi.org/10.3311/CCC2019-088

[3] H. Zhang et al, 2014 - Hainan Zhang, Shuangquan Shao, Hongbo Xu, Huiming Zou, Changqing Tian - "Free cooling of data centers: A review" Renewable and Sustainable Energy Reviews 35 (2014) 171-182

[4] Capozzoli and Primiceri, 2015 - Alfonso Capozzoli, Giulio Primiceri - "Cooling systems in data centers: state of art and emerging technologies" Energy Procedia 83 (2015) 484- 493 https://doi.org/10.1016/j.egypro.2015.12.168

[5] Li and Kandlikar, 2016 - Zhen Li And Satish G. Kandlikar - "Current Status and Future Trends in Data-Center Cooling Technologies" (Heat Transfer Engineering, 36:523-538, 2015) https://doi.org/10.1080/01457632.2014.939032

[6] K. Ebrahimi et al, 2014 - Khosrow Ebrahimi, Gerard F. Jones, Amy S. Fleischer - "A review of data center cooling technology, operating conditions and the corresponding low-grade waste heat recovery opportunities" - Renewable and Sustainable Energy Reviews 31(2014) 622-638 https://doi.org/10.1016/j.rser.2013.12.007

[7] Coles and Herrlin, LBNL 2016 - Henry Coles and Magnus Herrlin - "Immersion Cooling of Electronics in DoD Installations" May 2016 Lawrence Berkeley National Laboratory

[8] Longbottom, Techtarget 2013 - https://searchdatacenter.techtarget.com/feature/Modern-data-center-cooling-systems-No-muss-no-fuss-no-waste [9] H. Endo et al, 2014 - Hiroshi Endo, Hiroyoshi Kodama, Hiroyuki Fukuda, Toshio Sugimoto, Takashi Horie, Masao Kondo - "Effect of climatic conditions on energy consumption in direct fresh-air container data centers" - Sustainable Computing: Informatics and Systems 6 (2015) 17-25 https://doi.org/10.1109/IGCC.2013.6604472

[10] V. Mulay - Facebook, 2018 - https://code.fb.com/data-center-engineering/statepoint-liquid-cooling-system-a-new-more-efficient-way-tocool-a-data-center/

[11] Google Data Centers - https://www.google.com/about/datacenters/efficiency/internal/

[12] Uptime Institute, 2014 - https://journal.uptimeinstitute.com/2014-data-center-industry-survey/

[13] EPA, 2007 - https://www.energystar.gov/ia/partners/prod_development/downloads/EPA_Datacenter_Report_Congress_Final1.pdf

[14] G.A. Brady et al, 2013 - Gemma A. Brady, Nikil Kapur, Jonathan L. Summers, Harvey M. Thompson - "A case study and critical assessment in calculating power usage effectiveness for a data center" - Energy Conversion and Management 76 (2013) 155-161 https://doi.org/10.1016/j.enconman.2013.07.035

[15] Yuventi and Mehdizadeh, 2013 - "A critical analysis of Power Usage Effectiveness and its use in communicating data center energy consumption" Jumie Yuventi, Roshan Mehdizadeh - Energy and Buildings Journal 64 (2013) 90-94 https://doi.org/10.1016/j.enbuild.2013.04.015

[16] Avelar et al, 2012 - The Green Grid, 2012 https://www.thegreengrid.org/en/resources/library-and-tools/20-PUE\%3A-AComprehensive-Examination-of-the-Metric

[17] Horner and Azevedo, 2016 - Nathaniel Horner, Inês Azevedo "Power usage effectiveness in data centers: Overloaded and underachieving" - The Electricity Journal 29 (2016) 61-69 https://doi.org/10.1016/j.tej.2016.04.011

[18] Saunders et al, 2012 - "Research Methods for Business Students" 2012 - Mark N.K. Saunders, Philip Lewis, Adrian Thornhill ISBN-10: 9780273750758 